\title{
An Efficient Metaheuristic Approach for the Multi- Period Technician Routing and Scheduling Problem
}

\author{
Abouliakdane Khattara \\ Electrical Engineering Department, \\ Ferhat Abbas Setif 1 University, \\ Setif, Algeria \\ yekdane87@univ-setif.dz
}

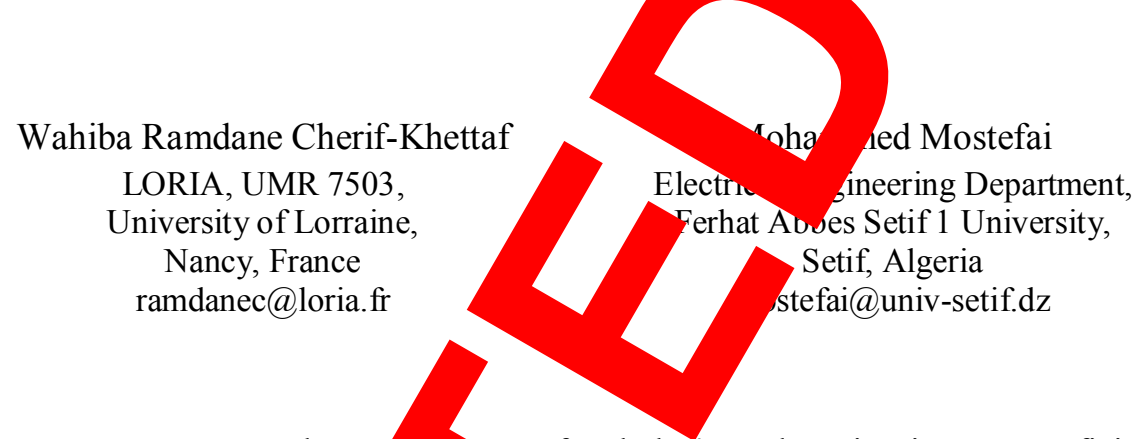

\begin{abstract}
In this paper, we address a new variant of the MultiPeriod Technician Routing and Scheduling Problem. This problem is motivated by a real-life industrial application in a telecommunication company. It is defined by a set of technicians having distinct skills that could perform a set of geographically scattered tasks over a multi-period horizon. Each task is subject to time constraints and must be done at most once over the horizon by one compatible technician. The objective is to minimize the total working time (composed by routing time, service time, and waiting time), the total cost engendered by the rejected tasks, and the total delay. Two variants of variable neighborhood descent are proposed, and three variants of
\end{abstract} variable neighborhood search to solve this pr Computational experiments are conducted on ben. instances from the literature. An analysis of the performan the proposed local search procedures is given. The results sh that our methods outperform the results of a mimetic metho published in the literature.

Keywords-technician routing and scheduling variable neighborhood search (VNS); descent (VND)

\section{INTRODUCTI}

Technician routing and schedulin froblem (TRS, a new challenge in logistics for the se ctor and especially for utility companies in e gy electricity), telecommunications, and water ontribution [1]. The TRSP consists of planning tas assigned to con ercial or technical personnel, over a set eriods (days) in order to visit industrial facilities or custom for d ent types of activities: installation, inspection, rep and r tenan Until recently, the TRSPs, both static a case ave received a limited attention. Thus, tho her $g$ publications and scientific reports is limited, altho al variants of the TRSP have been stur literatur hese variants can be divided into two cl es: ( 1 ne period TRSP, and (ii) multiperiod TRSP. T one $p$ has been studied by authors who con con ats as skills, time windows, tools, spare parts, stocm vice and stochastic travel times, multiple depots, and on priority [1-6]. For the multiperiod TRSP, we can mentio , which introduces the multiperiod technician scheduling problem with experience based on service times and stochastic customers. The aim is to minimize the exr horiz Ain ulti-period TRSP was proposed in 2007 [8]. This oblem co, in computing a schedule for technicians to perform a set of s on a five day horizon. The routing is not considered, and tasks have different proficiency 11 level constraints, that require a team of technicians. Authors in $]$ studied the one-periodic variant of this roblem, nam the service technician routing and scheduling blem by $t$ g on consideration the routing aspect. Authors h. ed a multi-period technician routing problem facea water distribution and treatment company. In [9], requests were divided into two categories (users requested ons and company scheduled visits), and the skill its were not included.

In this paper, we propose the study of a new multi-period TRSP variant where skill constraints and routing aspects are nsidered simultaneously, inspired by a realistic application in ie telecommunication field. From the above survey, it appears that most papers on TRSP considered several realistic constraints, but to the best of our knowledge, the multi-periodic variant of TRSP with skill constraints and routing aspects has not been considered in the literature. The papers that consider a multi-periodic TRSP with skill constraints, and routing aspects, included other specific constraints as the technician team constraint [10]. Our study is also an extension of the problem studied in $[9,11,12]$ in which the skill constraints are ignored. As the considered problem is NP-hard and since it results from the combination of complex constraints, large instances can hardly be solved by exact methods. So, the best way to tackle this problem is by using the metaheuristic approaches. We choose a variable neighborhood search (VNS) to solve our problem, because its effectiveness has been proven on a number of variants of vehicle routing problems (VRP) as the vehicle routing problem with time windows [13], the vehicle routing problem with multiple depots and time windows [14, 15], the periodic vehicle routing problem [16], and the workforce scheduling and routing problem [17].

In this paper, we propose two variants of variable neighborhood descent, as well as three variants of variable neighborhood search to solve the TRSP with skill constraints and routing aspects. 


\section{PROBLEM DESCRIPTION}

We consider a multi-period horizon $H$ of several days (typically one week). For each day $h \in H$, a set of technicians $K$ with different skills are available (a technician has one skill or more). Each technician $k \in K$ has a known starting and ending location $d \in D$, which corresponds to the technician's home or office (the starting location is the same with the ending location). Each technician has a working time limit per day Maxtimek, $h$. Requests belong to two categories: non-urgent tasks (NT) generated by the company, and urgent requests (UT) formulated by customers through a call center, for emergency reasons. Note that $U T \cup N T=T$, with $T$ the set of all tasks known in advance. Let $s i$ be the service time of the task $i$. The urgent tasks $i \in U T$ are planned on a fixed day $h i$ and are subjected to customer appointments within a given time window $(b i, e i)$, where $b i$ is the beginning of the time window, and $e i$ the end of the time window. The task $i$ could be affected to a technician $k$ if the arriving time at task $i$ (denoted aik) is before the end of the time windows $(a i k<e i)$. If $\forall k \in K, a i k \geq e i$, then the task $i$ is considered as a rejected task. If $a i k \leq b i$, a waiting time $W i k$ occurs, with Wik=bi-aik. If $a i k \leq e i$, and $a i k+s i>e i$, a delay $L i k$ occurs, with $L i k=(a i k+s i)-e i$. Non-urgent tasks are characterized by a validity period composed of one or several days $[h b i, h e i] \in H$, where $h b i$ is the early day and hei is the deadline for the execution of $i$. A request $i$ requires certain skills (qualifications), and must be executed by only one compatible technician. The goal is to build a set of routes ner day and per technician (at most $|K h|$ routes per day). Eac $R h k$ is a sequence of tasks assigned to only one techn and one-day $h$. The following constraints must be satisfiea each task must be executed at most once within the valid period or within the time window, 2) the total time of each route $R h k$ should not exceed Maxtimek, $h, 3)$ requirements must be respected, 4) each rou end at the same location $d \in D$. The measured in monetary units denoted minimizing three costs: (i) the total working routing time that depends on the number by each technician, the service time a the total cost engendered by the eject delay.

\section{SOLUTIONMETHODOLOGY}

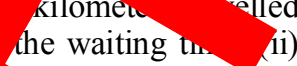
$v_{\text {s. and }}$, iii) the total

In this section, we descril ne general framework of the variable neighborhood searc NS d then we present the basic components of the $\mathrm{V}$ that $y$ ave doped to solve our problem.

\section{A. Variable Neighborhood Sear}

VNS is a metahe 19] for approximate combinatorial and n-line optimization problems $[20,21]$. VNS bas systematic changes of neighborhood stru fing the search for a (near) optimal solution of a consider descent phase, to improm solution, and shaking and perturbation phase that aims to escape local optima traps. The main structure of the VNS (Algorithm 1) is shown in Figure 1.

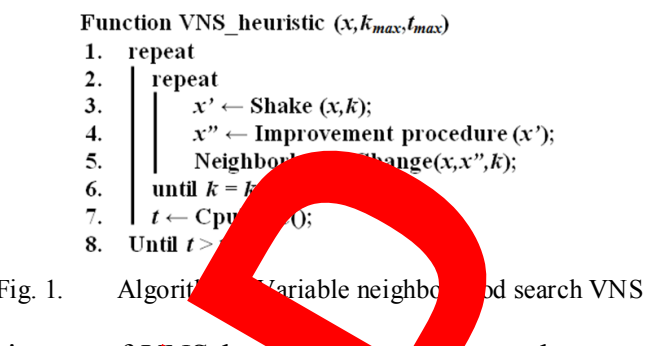

The inputs of Y's heurm $\max _{\max }$ and $t_{\max }$, and they present the initial ation, the num of neighborhoods to be explored and $t^{1}$ maxiy allowed CPU time. The main ingredients of ariab neight hood search include an improvement $\mathrm{dv}$ imp the current solution and a shaking pracedu. sturb search and escape from the correspon valley, 3 and 4. The improvement procedu line 5 could single local search or an ordered list of aighborhoods.

B.

We propose to is an initial solution the best insertion with sorting list. This method is performed by two ps. In the first step, a list of unserved tasks $(L, L=T)$, is orted in in ing order according to validity day $(V D)$, that epresents the igth of the period (number of days) in which tasks can done, $V D_{i}=h e_{i}-h b_{i}$. In the second step, the t a task $i$ from the head of $L$, and scan all feas. The insertion cost of $i$ in a route $R_{h k}$ between two tasks $x$ and $y$, named $\delta\left(i, R_{h k}, x, y\right)$ will be in (1). The algorithm performs the best insertion.

$$
\begin{aligned}
(i, R h k, x, y) & =C_{x i}+C_{i y}-C_{x y}+\Sigma(j \in R h k \cup i) W_{j k} \\
& +\Sigma(j \in R h k \cup i) L_{j k}
\end{aligned}
$$

\section{Local Search Procedures}

We propose five local search operators to be used either individually or together to focus the search in the inner loop of VNS. We consider three intra-route and two inter-route local search methods. The best improvement strategy is used for each method. The local search methods are:

- One intra-route relocate: one node (task) from the route is removed and reinserted in other positions in the same route.

- One intra-route exchange: two nodes (tasks) are exchanged in the same route.

- 2 opt: two arcs are removed and reinserted in the same route

- One inter-route relocate: one node (task) from the route is removed and reinserted in one other route in the solution.

- One inter-route exchange: two nodes (tasks) are exchanged between two different routes.

\section{Variable Neighborhood Descent Procedures}

The variable neighborhood descent (VND) procedures explore several neighborhood structures either in a sequential or nested (or composite) fashion to possibly improve a given solution [21] because the solution which is a local optimum with respect to several neighborhood structures is more likely 
to be a global optimum than the solution generated as a local optimum for just one neighborhood structure. The order of neighborhoods may play an important role in the quality of the final solution [22]. Two variants of VND are discussed in this paper regarding the decision made in neighborhood change procedure. If an improvement has been detected in some neighborhood: (1) Basic VND (B-VND): we return to the first neighborhood on the list, (2) Union VND (U-VND): at each iteration all the neighborhoods in the list are used to explore the search, and the next incumbent solution is the best one found by the best neighborhood. The outline of basic VND is presented in Algorithm 2 (Figure 2). The steps of the sequential neighborhood change which is presented in line 5 (Algorithm 1) and line 7 (Algorithm 2) are given in Algorithm 3 (Figure 3). If an improvement of the incumbent solution in some neighborhood structure occurs, the search is resumed in the first neighborhood structure (according to the defined order) of the new incumbent solution, otherwise the search is continued in the next neighborhood (according to the defined order).
Finally, we compare and evaluate the VNS procedures proposed in this paper.

\section{A. Description of Experimental Data Sets}

In order to evaluate a the performance of the proposed approaches, $\mathrm{w}$ ompan $\mathrm{n}$ with the methods proposed by Tricoire in 1,12$]$. Thu tances of $[9,11,12]$ are used for tests. For the skill con ints in our problem are relaxed, and lunch onstrain re added. They are inspired by a real li case, an vailable with detailed experimental res in [9] as as on the web site: http://www. em z-au Juting-pbs/. All instances have a five-day plann horiz and thr technicians available every day. The den domly stributed over a $40 \mathrm{~km}^{2}$ map, and Euclidean a. are $a$. The technicians drive at a constant a age spea $\mathrm{a} / \mathrm{h}$. Two sizes of instances are tested $\mathrm{C}$ ith 100 custom and $\mathrm{C} 2$ with 180 customers, each one y variants according to the distribution and the perce ge windows and the percentage of urgent and non- gent tasks.

\section{B $\quad$ luation Of The Performance Of The Local Search}

We stud e impact of the locale search procedures. The esults are si $n$ in Table I. The first column indicates the ne of each stance. Column 2 presents the result found by ation, which is based on the best insertion stratus. remained columns provide both the Gap and the computing time for each local search operator. The Gap is

by (2). Row 13 mentions the average results of all The ranks of the local search operators according to eir performances and computing time are provided in the last wo rows. The best results are in bold.

Fig. 2. Algorithm 2: Variable neighborhood descent VND

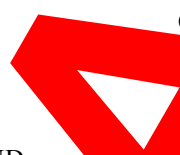

Gap\% $=\left(f(x)_{\text {heuristic }}-f(x)_{\text {heuristic }+L S}\right) / f(x)_{\text {heuristic }+L S}$

From Table I, we note that the 2 opt operator is the best one in almost all instances but it is the third one in terms of computing time. It is also worth noting that all the operators perform well and they all improve on average at least $7.14 \%$ and at most $9.66 \%$ the results found by the heuristic.

\section{Variable Neighborhood Descent Procedures}

The aim of this section is to evaluate and to compare different variants of VND procedures according to the manner in which the neighborhood is changed after each improvement occurs. Namely, it is obvious that the order of neighborhoods on the list affect the performance of VND procedures [22]. Thus, we take into account two possible orders of LS procedures according to their performance: 1) the value of $f(x)$, and 2) the computing time. The used orders are mentioned in Table II. The results of VND procedures on Tricoire instances are summarized in Table III. The settings of the VND variant are provided in column and row headings as described above. For example, in Table III, the values in the two cells, at the intersection of the row C100_1 and $4^{\text {th }}$ column, correspond to value achieved by B-VND that explores neighborhoods using the 1 st order, as well as its execution time in second. The next column reports the percentage deviation of the obtained solution compared to best solution of the mimetic algorithm 
proposed by Tricoire $[9,11,12]$. The deviations are calculated by:

$$
\operatorname{Dev} \%=\left(f(x)_{V N D}-f(x)_{\text {memetic }}\right) / f(x)_{\text {memetic }}
$$

The next column reports the percentage deviation of the obtained solution compared to the result found by the initial solution, which is calculated by (2). In Table III, we report the results obtained by B-VND and U-VND using the two proposed orders. The average results are mentioned in the two last rows. In Table III, values in bold followed by a star represent new best solution

TABLE I. COMPARISON BETWEEN LOCAL SEARCH PROC

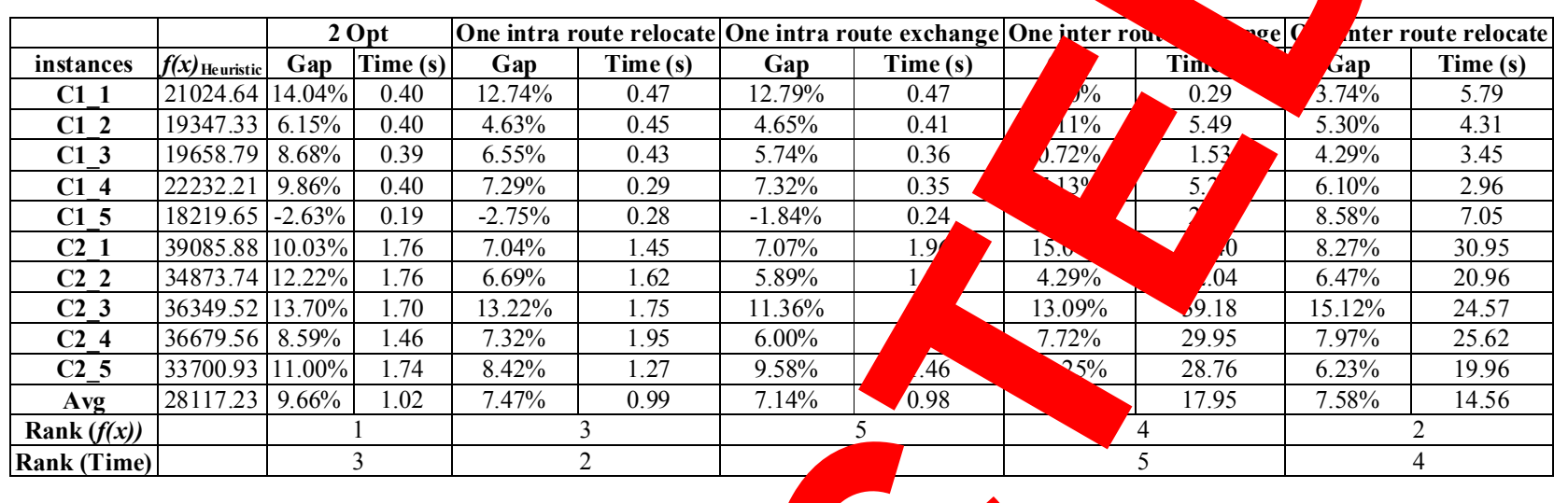

TABLE II. ORDERS OF LOCAL SEARCH PROCEDURES

\begin{tabular}{|c|c|c|c|}
\hline \multicolumn{2}{|c|}{ 5 Local search operators } & 1st & 2nd \\
\hline \multirow{4}{*}{$\begin{array}{c}\text { Order of } \\
\text { local search }\end{array}$} & 2 Opt & 1 & 3 \\
\cline { 2 - 4 } & One intra route relocate & 3 & 2 \\
\cline { 2 - 4 } & One Intra route exchange & 5 & 1 \\
\cline { 2 - 4 } & One Inter route exchange & 4 & 5 \\
\cline { 2 - 4 } & One inter route relocate & 2 & 4 \\
\hline
\end{tabular}

From the results presented in Table III, we may concl. the following: The VND variants that explore neighborhoods 1 $1^{\text {st }}$ order offer the best results in both objective ion and

CPU time co red to the other order type. If we consider the verage resul ver all test instances, it appears that the best ore resy abtained by U-VND even when we change thu sighborhoods. So we can say that the U-VND is more efrective than B-VND in terms of the objective function and CPU Time. From the average results over all test instances that all our VND procedures implemented and cussed in this paper are competitive and perform better than ne mimetic algorithm when solving the same problem. For 6 instances among 10, a new best solution is found by our thod.

\begin{tabular}{|c|c|c|c|c|c|c|c|c|c|c|c|c|c|c|}
\hline & & & & & & & & & \multicolumn{6}{|c|}{ U-VND } \\
\hline & & Orders & & $1 \mathrm{st}$ & & \multicolumn{3}{|c|}{ 2nd } & \multicolumn{3}{|c|}{$1 \mathrm{st}$} & \multicolumn{3}{|c|}{ 2nd } \\
\hline Instances & & $\begin{array}{c}\text { Mimetic of } \\
\text { Tricoire }\end{array}$ & Value & $\begin{array}{l}\text { \% De } \\
\text { mime }\end{array}$ & & & $\begin{array}{l}\% \text { Dev } \\
\text { mimetic }\end{array}$ & $\begin{array}{c}\text { \% Dev } \\
\text { heuristic }\end{array}$ & Value & $\begin{array}{c}\text { \% Dev } \\
\text { mimetic }\end{array}$ & $\begin{array}{c}\text { \% Dev } \\
\text { heuristic }\end{array}$ & Value & $\begin{array}{c}\% \text { Dev } \\
\text { mimetic }\end{array}$ & $\begin{array}{c}\text { \% Dev } \\
\text { heuristic }\end{array}$ \\
\hline \multirow{2}{*}{ C100_1 } & $f(x)$ & 17893.91 & 17578.37* & & $19.61 \%$ & 17594.18 & $-1.68 \%$ & $19.50 \%$ & $17578.37 *$ & $-1.76 \%$ & $19.61 \%$ & 17594.03 & $-1.68 \%$ & $19.50 \%$ \\
\hline & Time (s) & & 9.92 & & & 11.42 & & & 8.97 & & & 13.28 & & \\
\hline \multirow{2}{*}{ C100_2 } & $f(x)$ & 15977.12 & 17202.92 & & & 17136.03 & $7.25 \%$ & $12.90 \%$ & 17153.67 & $7.36 \%$ & $12.79 \%$ & 17164.61 & $7.43 \%$ & $12.72 \%$ \\
\hline & Time (s) & & 9.52 & & & 9.58 & & & 8.06 & & & 8.73 & & \\
\hline \multirow{2}{*}{ C100_3 } & $f(x)$ & 16714.03 & 17493.5 & $4.66 \%$ & $12.38 \%$ & $\sqrt{529.38}$ & $4.88 \%$ & $12.15 \%$ & 17491.94 & $4.65 \%$ & $12.39 \%$ & 17538.11 & $4.93 \%$ & $12.09 \%$ \\
\hline & Time $(\mathrm{s})$ & & & & & 5.93 & & & 5.03 & & & 6.74 & & \\
\hline \multirow{2}{*}{ C100_4 } & $f(x)$ & 17489.36 & & 4. & $21.58 \%$ & 18265.73 & $4.44 \%$ & $21.72 \%$ & 18229.85 & $4.23 \%$ & $21.95 \%$ & 18031.33 & $3.10 \%$ & $23.30 \%$ \\
\hline & Time (s) & & & & & 12.68 & & & 9.5 & & & 14.03 & & \\
\hline \multirow{2}{*}{ C100_5 } & $f(x)$ & 16025.91 & & & 0 & 16611.1 & $3.65 \%$ & $9.68 \%$ & 16535.47 & $3.18 \%$ & $10.19 \%$ & 16364.41 & $2.11 \%$ & $11.34 \%$ \\
\hline & Time (s) & & & & & 9.78 & & & 9.91 & & & 15.47 & & \\
\hline \multirow{2}{*}{ C180_1 } & $f(x)$ & 28945.36 & & & $.63 \%$ & 29299.56 & $1.22 \%$ & $33.40 \%$ & $28405.93 *$ & $-1.86 \%$ & $37.60 \%$ & 28579.51 & $-1.26 \%$ & $36.76 \%$ \\
\hline & Time (s) & & & & & 107.17 & & & 84.19 & & & 96.87 & & \\
\hline \multirow{2}{*}{ C180_2 } & $f(x)$ & 31191.1 & & $-9.73 \%$ & $23.86 \%$ & 27780.88 & $-10.93 \%$ & $25.53 \%$ & 27748.3 & $-11.04 \%$ & $25.68 \%$ & 27729.19* & $-11.10 \%$ & $25.77 \%$ \\
\hline & Time $(\mathrm{s})$ & & 66 & & & 72.82 & & & 58.22 & & & 46.09 & & \\
\hline \multirow{2}{*}{ C180_3 } & $f(x)$ & 2777 & 264 & 6 & $37,35 \%$ & 27472,29 & $-0,92 \%$ & $32,31 \%$ & $26034,96 *$ & $-6,11 \%$ & $39,62 \%$ & 26886,39 & $-3,04 \%$ & $35,20 \%$ \\
\hline & Time $(\mathrm{s})$ & & & & & 89,47 & & & 78,46 & & & 77,85 & & \\
\hline \multirow{2}{*}{ C180_4 } & $f(x)$ & 3024 & 48,92 & $-2,96 \%$ & $24,98 \%$ & 29522,29 & $-2,39 \%$ & $24,24 \%$ & 30124,57 & $-0,40 \%$ & $21,76 \%$ & 29238,94* & $-3,33 \%$ & $25,45 \%$ \\
\hline & Time (s) & & & & & 76,22 & & & 52,22 & & & 61,71 & & \\
\hline \multirow{2}{*}{ C180_5 } & $f(x)$ & 28158,57 & 00 & $-4,54 \%$ & $25,37 \%$ & 26566,25 & $-5,65 \%$ & $26,86 \%$ & 26395,74* & $-6,26 \%$ & $27,68 \%$ & 26625 & $-5,45 \%$ & $26,58 \%$ \\
\hline & Time (s) & & 78,74 & & & 93,93 & & & 70,71 & & & 60,92 & & \\
\hline \multirow{2}{*}{ Average } & $f(x)$ & 23036,94 & 22655,33 & $-1,66 \%$ & $24,11 \%$ & 22777,77 & $-1,13 \%$ & $23,44 \%$ & 22569,88 & $-2,03 \%$ & $24,58 \%$ & 22575,15 & $-2,00 \%$ & $24,55 \%$ \\
\hline & Time $(\mathrm{s})$ & & 44,96 & & & 48.9 & & & 38.53 & & & 40.17 & & \\
\hline
\end{tabular}


It is worth noting that all VND procedures also perform better and they all improve on average at least $23.44 \%$ and at most $24.58 \%$ the results found by the heuristic. That means that VND procedures improve the solution in average $15 \%$ more than the single local search operators (Table I).

\section{Variable Neighborhood Search Procedures}

In this section we evaluate and compare three variants of VNS procedures regarding to the improvement procedures in the inner loop: (1) B-VNS that uses a simple local search in the inner loop at each iteration and move to the other one as in Algorithms 1 and 3, (2) VNS_B-VND that uses a B-VND procedure, and (3) VNS U-VND that uses a U-VND in the improvement phase. The neighborhoods in the VND procedures are ordered in ar. The performances of VNS procedures have be ested o $\mathrm{C} 1$ of the instances described above. We to d our VNS cedures by using 4 different time limits, ing from 36 o 1140 s. For each instance and each VNS the f rithm 5 times. The results are summari in Tab. gure 4.
For each time limit and each VNS variant, we report the deviation from the best solution found over all variants of instance of class $\mathrm{C} 1$ in 5 runs compared to the best solution found by the mimetic algorithm of $[9,11,12]$ (named $\%$ Dev Best in Table IV). We also compute the deviation from the average value of the solutions found in 5 runs for all instances of $\mathrm{C} 1$ compared to the best solution found by the mimetic algorithm (named \% Dev Avg in Table IV). The deviations are calculated by (3).

TABLE IV. EVALUATION OF DIFFERENT VARIA

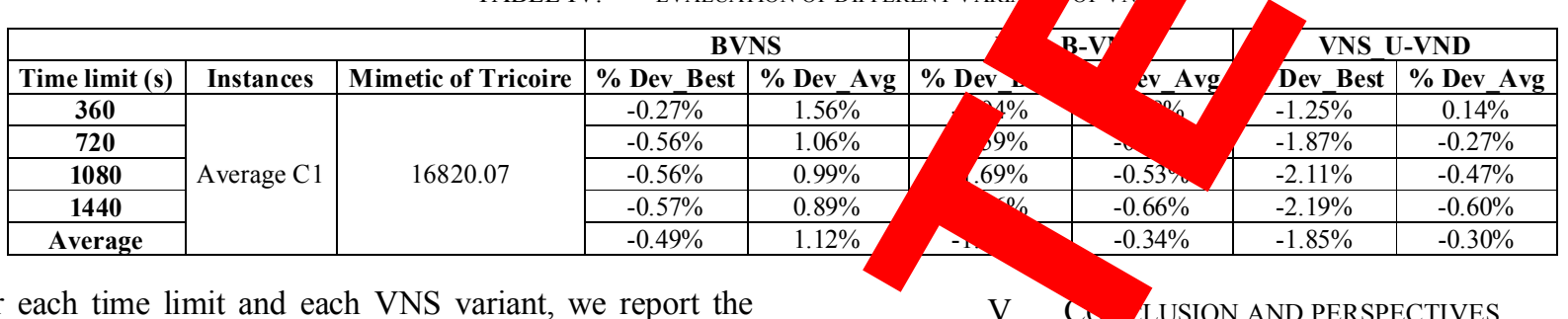

V. Co LUSION AND PERSPECTIVES

this paper, we considered a new variant of the Multieriod Techp ian Routing and Scheduling Problem motivated by a real-lit dustrial application in a Telecommunication ompany. T olve the problem, two variants of variable shorhor escent B-VND and U-VND, as well as three a. able neighborhood search BVNS, VNS_B-VND and VNS_U-VND are proposed. All heuristic methods were anted and compared with the methods proposed by Tricoire $[9$, he results confirm the effectiveness of our methods. garding future work, we will generate other instances to atensify the experimentations. Also, we will consider the dynamic aspect, where the demands appear dynamically over planning horizon.

\section{REFERENCES}

[1] V. Pillac, C. Gueret, A. L. Medaglia, "A parallel matheuristic for the technician routing and scheduling problem", Optimization Letters, Vol. 7, No. 7, pp. 1525-1535, 2013

[2] J. Xu, S. Y. Chiu, "Effective heuristic procedures for a field technician scheduling problem", Journal of Heuristics, Vol. 7, No. 5, pp. 495-509, 2001

[3] E. Hadjiconstantinou, D. Roberts, "Routing under uncertainty: an application in the scheduling of field service engineers", in: The vehicle Routing Problem, pp. 331-352, Society for Industrial and Applied Mathematics, 2001

[4] E. Delage, Re-optimization of Technician Tours in Dynamic Environments with Stochastic Service Time, Ecole des Mines de Nantes, 2010

[5] C. E. Cortes, F. Ordonez, S. Souyris, A. Weintraub, "Routing technicians under stochastic service times: a robust optimization approach", TRISTAN VI: The Sixth Triennial Symposium on Transportation Analysis, Phuket Island, Thailand, June 10-15, 2007

[6] V. Pillac, C. Gueret, A. Medaglia, "On the dynamic technician routing and scheduling problem", 5th International Workshop on Freight Transportation and Logistics, Mykonos, Greece, May 21-25, 2012

[7] X. Chen, B. W. Thomas, M. Hewitt, "Multi-Period Technician Scheduling with Experience-based Service Times and Stochastic Customers", Computers and Operations Research, Vol. 82, No. C, pp. 114,2017

[8] W. Jaskowski, ROADEF Challenge 2007: Technicians and Interventions Scheduling for Telecommunications, Poznan University of Technology, 2007 
[9] F. Tricoire, Optimisation de Tournees de Vehicules et de Personnels de Maintenance: Application a la Distribution et au Traitement des Eaux, PhD Thesis, Universite de Nantes, 2006 (in French)

[10] A. A. Kovacs, S. N. Parragh, K. F. Doerner, R. F. Hartl, "Adaptive large neighborhood search for service technician routing and scheduling problems", Journal of Scheduling, Vol. 15, No. 5, pp. 579-600, 2012

[11] N. Bostel, P. Dejax, P. Guez, F. Tricoire, "Multiperiod planning and routing on a rolling horizon for field force optimization logistics", in: The Vehicle Routing Problem: Latest Advances and New Challenges, pp. 503-525, Springer, 2008

[12] F. Tricoire, N. Bostel, P. Dejax, P. Guez, "Exact and hybrid methods for the multiperiod field service routing problem", Central European Journal of Operations Research, Vol. 21, No. 2, pp. 359-377, 2013

[13] O. Braysy, "A reactive variable neighborhood search for the vehicle routing problem with time windows", INFORMS Journal of Computing, Vol. 15, No. 4, pp. 347-368, 2003

[14] M. Polacek, R. F. Hartl, K. Doerner, M. Reimann, "A variable neighborhood search for the multi depot vehicle routing problem with time windows", Journal of Heuristics, Vol. 10, No. 6, pp. 613-627, 2004

[15] S. Salhi, A. Imran, N. A. Wassan, "The multi-depot vehicle routing problem with heterogeneous vehicle fleet: Formulation and a variable neighborhood search implementation", Computers \& Operations Research, Vol. 52B, pp. 315-325, 2014

[16] M. Elbek, S. Wohlk, "A variable neighborhood search for the multiperiod collection of recyclable materials", European Journal of Operational Research, Vol. 249, No. 2, pp. 540-550, 2016

[17] R. L. Pinheiro, D. Landa-Silva, J. Atkin, "A variable neighbourhood search for the workforce scheduling and routing problem", in: Advances in Nature and Biologically Inspired Computing, pp. 247-259, Springer, 2016

[18] N. Mladenovic, P. Hansen, "Variable neighborhood search", Computers \& Operations Research, Vol. 24, No. 11, pp. 1097-1100, 1997

[19] P. Hansen, N. Mladenovic, "Variable neighborhood search: and applications", European Journal of Operational Research, No. 3, pp. 449-467, 2001

[20] P. Hansen, N. Mladenovic, J. A. M. Perez, "Variable neighbourh search: methods and applications", Annals of Operations Research, Vo 175, No. 1, pp. 367-407, 2010

[21] P. Hansen, N. Mladenovic, R. Todosijevic, S neighborhood search: basics and variants", Computational Optimization, Vol. 5, No. 3, pp. 1-

[22] A. Mjirda, R. Todosijevic, S. Hanafi, P. "Sequential variable neighborhood descent varian on the traveling salesman problem", Int tional Operational Research, Vol. 24, No. 3, pp. $6 \quad 33,2016$
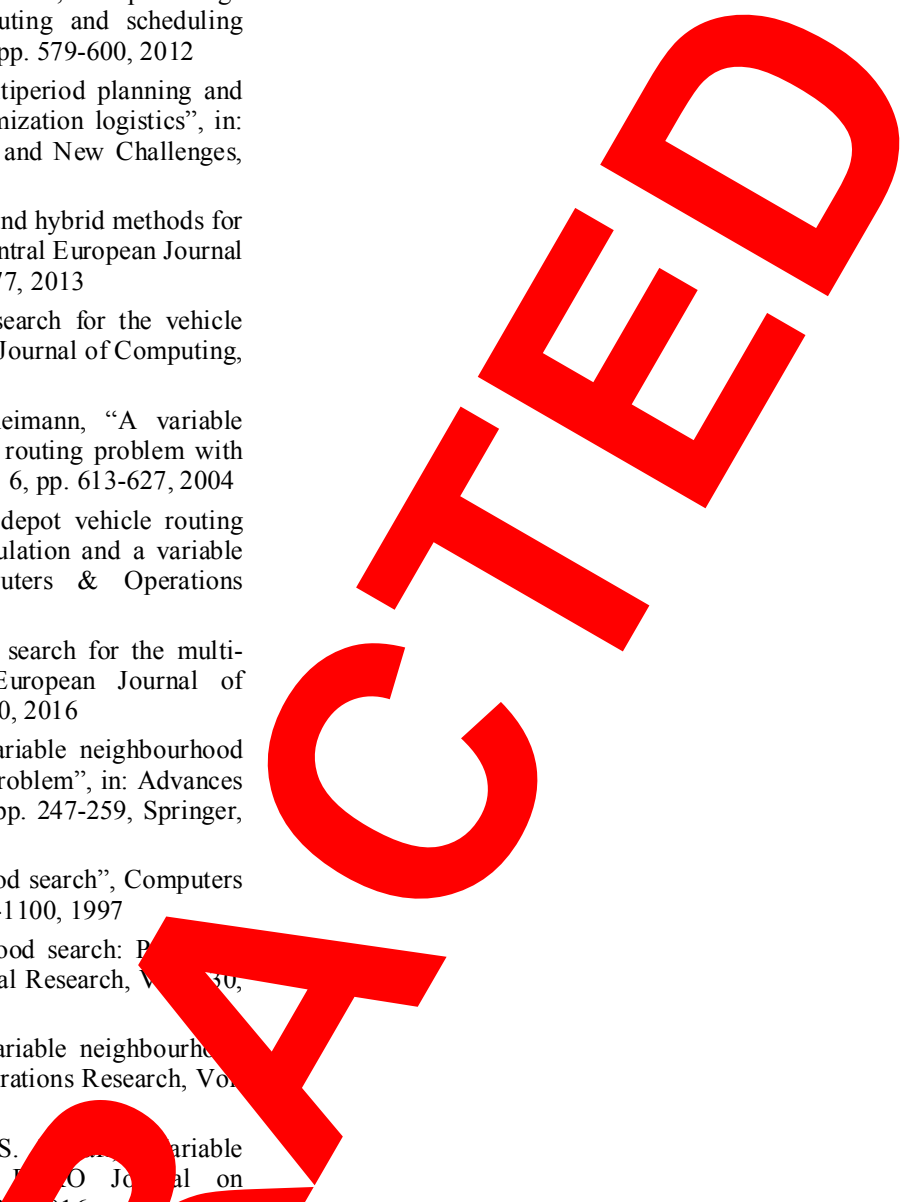
${ }_{016} \mathrm{~J}$ 016

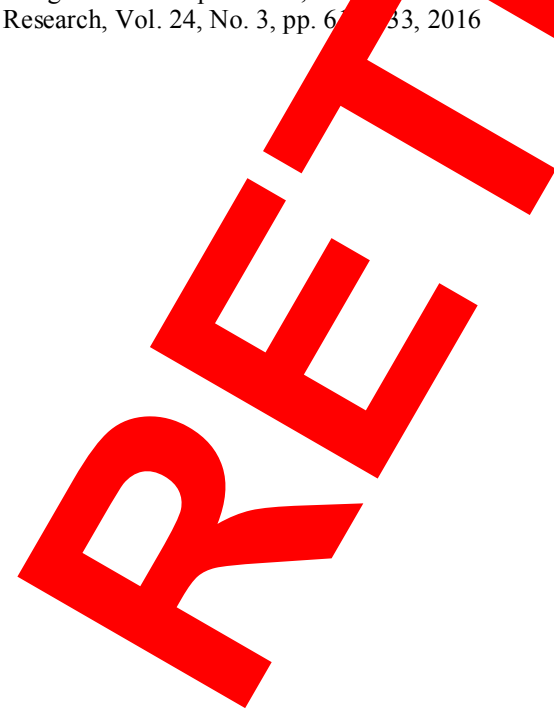

